\section{Discursos de género de las mujeres emprendedoras por oportunidad. El caso español.}

Gender discourses of women entrepreneurs by opportunity. The Spanish case.

IGNASI BRUNET*

Alejandro PIzZ|*

\section{Resumen}

Se presentan los resultados de una investigación sobre emprendedoras en España. Se analizan los discursos de género de las mujeres emprendedoras por oportunidad. Específicamente, se estudia la relación entre el discurso de la conciliación familiar, por un lado, y el de la liberación de "responsabilidades

Universidad Rovira i Virgili. España. Correo electrónico: ignasi. brunet@urv.cat.

** Universidad de Valencia. España. Correo electrónico: alejandro. pizzi@uv.es. domésticas/reproductivas", por otro, entre las emprendedoras por oportunidad.

La muestra cualitativa está conformada por mujeres (y madres) profesionales autónomas/ empresarias del sector servicios, de elevado capital cultural. Se llevaron a cabo 40 entrevistas en profundidad y 11 grupos de discusión. Sobre la base del análisis de discurso, se elabora una tipología de emprendedoras por oportunidad, según los discursos relacionados con el trabajo "productivo" y "reproductivo". Como conclusión, se comprueba la existencia de un imaginario social "masculinizado" entre las emprendedoras, que habilitan dos lógicas de comportamiento: aquellas que dan prioridad a la vida laboral, por un lado, y aquellas que buscan conciliar la vida laboral y familiar.

Palabras clave: trabajo productivo; trabajo reproductivo; género; división sexual del trabajo; comportamiento emprendedor

\section{Abstract:}

This article presents the results of research on women entrepreneurs in Spain. The focus of the analysis is on women's discourse on gender. More specifically, the article studies the relationship between discourses on family compatibility and on the release of "domestic / reproductive responsibilities " between entrepreneurs by opportunity. The qualitative sample is formed by women (and mothers) autonomous professionals / entrepreneurs from the services sector, where 40 in-depth interviews and 11 focus groups were conducted. Based on the analysis of discourses, a typology of entrepreneurial opportunity resulted, according 
to the arguments related to the "productive" and "reproductive" work. The study shows the existence of a "masculinized" social imaginary among woman entrepreneurs, which enables two logics of behaviour: those that give priority to working life, on the one hand, and those seeking to reconcile work and family life.

Key words: productive work, reproductive work, gender, sexual division of work, entrepreneur behavior.

\section{Introducción}

Este artículo reelabora una parte de los resultados de una investigación de mayor aliento sobre género y creación de empresas en España (Brunet et al. 2009a, 2009b, 2011; Brunet y Pizzi, 2011). Concretamente, en este artículo reelaboramos y profundizamos el análisis de los perfiles de las mujeres emprendedoras por oportunidad en diferentes regiones de España que se llevó a cabo en la investigación original (Brunet et al. 2009a). La investigación original completa trianguló un abordaje cuantitativo y cualitativo, y permitió establecer categorías analíticas (estadísticamente significativas) de mujeres emprendedoras. A continuación, se expusieron los discursos de género de los perfiles determinados previamente. En este artículo, concretamente, reelaboramos una parte de los datos cualitativos mediante la construcción de una tipología de "emprendedoras por oportunidad", que nos permite comprender en profundidad las motivaciones típicas y las lógicas de comportamiento emprendedor por parte de este perfil en diferentes Comunidades Autónomas de España. La investigación original se planteó por los siguientes motivos. En primer lugar, se consideró pertinente abordarla porque, a partir del Consejo Europeo de Amsterdam de 1997 (Comisión de las Comunidades Europeas, 2003), uno de los objetivos principales de la Estrategia Europea para el Empleo (EUE) es promover el espíritu empresarial, con aplicación a nivel español y europeo (Brunet et al. 2009a, 2009b, 2011). El argumento de la EUE es que el fomento del espíritu empresarial favorece la innovación, y ésta, a su vez, constituye uno de los factores principales para modernizar la economía. En segundo lugar, la literatura evidencia que los efectos de las diferencias de género sobre la creación de empresas constituyen un ámbito de investigación con cierta trayectoria internacional; sin embargo, constituye una laguna académica en España e Iberoamérica.

Los "emprendedores/as por oportunidad" son aquellas personas dotadas de suficientes recursos financieros y culturales que visualizan una oportunidad de mercado y desarrollan estrategias coherentes para aprovecharla. Los "emprendedores/as por necesidad" carecen de dichosrecursos, peroasumenunemprendimiento en condiciones precarias para construir un refugio frente al desempleo (Moriano, 2005). Por tanto, los "emprendimientos por oportunidad" son los que proveen una mayor aportación de valor a la economía, y las diferencias entre hombres y mujeres en este tipo de iniciativas justifican el conocimiento de los discursos del perfil de emprendedoras de oportunidad, ya que pueden constituir insumos importantes para elaborar políticas de promoción de la actividad emprendedora femenina.

El objetivo de este artículo es reconstruir y analizar los discursos que organizan las relaciones que las mujeres emprendedoras por oportunidad experimentan entre su profesión y 
sus actividades domésticas y familiares. Dichos discursos los reconstruimos desde el eje "trabajo productivo/reproductivo". Al respecto, la pregunta de investigación que estructura el análisis de discurso es si la opción por el autoempleo y el emprendimiento femenino radica en la flexibilidad que éste reporta a las mujeres, y que permite una mejor conciliación de la vida laboral y familiar, o bien si se fundamenta en la posibilidad, para las mujeres, de liberarse de las tareas reproductivas no reconocidas socialmente.

Luego de esta introducción se expone la metodología utilizada, y se desarrollan los conceptos centrales del marco teórico. Seguidamente se presenta el análisis reelaborado de los discursos típicos de las mujeres emprendedoras por oportunidad en España. Por último, se desarrollan las conclusiones y las líneas de investigación que quedan abiertas.

\section{Metodología de trabajo}

La recogida de los datos de la investigación original completa ya mencionada (de la que este artículo analiza sólo una parte de sus resultados) se efectuó en dos etapas. En una primera etapa, se ha examinado la dimensión cuantitativa del universo de mujeres emprendedoras en España, así como sus características sociodemográficas. Las fuentes de datos utilizadas fueron las bases estadísticas del Instituto Nacional de Estadística -España-, EUROSTAT, y el Proyecto Global Entrepreneurship Monitor). El análisis de estos datos, tanto en relación con las características de las mujeres como con las características de los emprendimientos creados por ellas, ha permitido construir dos perfiles de mujeres emprendedoras (que confirman los resultados de investigaciones anteriores): "emprendedoras por necesidad" y "emprendedoras de oportunidad" (Ardchivili et al. 2003; Acs et al. 2005).

En la segunda etapa de la investigación se describieron los discursos típicos de género de las emprendedoras de oportunidad. El objetivo de este artículo es, a partir de aquella descripción, reelaborar la información cualitativa mediante la construcción de una tipología de perfiles de emprendedoras por oportunidad en función del vínculo entre trabajo productivo/reproductivo. En especial, nos interesa la relación entre el discurso de la conciliación familiar, por un lado, y el de la liberación de "responsabilidades domésticas/reproductivas", por otro.

De acuerdo con los objetivos de investigación, la muestra cualitativa está conformada por mujeres (y madres) emprendedoras por oportunidad. Dado que nos interesa reconstruir los discursos sociales que vinculan la actividad profesional emprendedora con el trabajo reproductivo (y la maternidad), el perfil de las entrevistadas responde a profesionales autónomas/ empresarias del sector servicios, de elevado capital cultural, con edades comprendidas entre los 30 y 45 años. En la muestra seleccionada tienen diferente estado civil: casadas, en pareja de hecho, separadas, pero todas tienen hijos, dado nuestro objetivo de investigación.

Este análisis está basado en 40 entrevistas en profundidad a distintos perfiles de mujeres profesionales de diferentes comunidades autónomas, con el objetivo de conocer sus percepcionesy valoraciones individuales respecto a su desempeño profesional, su relación con la familia, sus expectativas de progreso laboral, y las principales dificultades y oportunidades que perciben. Analizamos 12 entrevistas a emprendedoras en Cataluña (Barcelona); 
10 a emprendedoras de Andalucía (Sevilla y Málaga), 10 a emprendedoras de Valencia; 8 a emprendedoras de Murcia. Los perfiles profesionales de las emprendedoras incluyeron diseñadoras de software, investigadoras de mercados y marketing, consultoras fiscales y financieras, empresarias de laboratorio químicos y farmacéuticos, empresarias inmobiliarias, consultoras de $\mathrm{RRHH}$, psicólogas, empresarias de sector turístico, abogadas, y periodistas free-lance. Aproximadamente la mitad de las entrevistadas (18) han montado su propia pequeña empresa y tienen empleados a cargo (hasta 10), y la otra mitad (22) son emprendedoras autónomas sin empleados a cargo.

Asimismo, se analizaron 11 grupos de discusión con mujeres (y madres) emprendedoras por oportunidad, profesionales, con el objetivo de provocar la emergencia de discursos sociales que den cuenta del vínculo entre actividad profesional y tareas "domésticas" y "familiares". Al respecto, se realizaron grupos de discusión en Cataluña (3), Andalucía (3), Valencia (3) y Murcia (2). El detalle de la composición de los grupos de discusión, de los que citamos fragmentos de conversaciones para ilustrar discursos, se referencian a pie de página durante el análisis. Sobre esta base hemos reelaborado y sistematizado los discursos sociales de las "emprendedoras de oportunidad", presentándolos en su tipicidad, es decir, de una manera internamente coherente. No estimamos la distribución cuantitativa de cada tipo entre las emprendedoras, sino que presentamos las características cualitativas de los discursos que vinculan emprendimiento y tareas familiares.

La interpretación de las entrevistas se basa en el Análisis Crítico del Discurso (Van Dijk 2000, 2009; Fairclough 2003), entendido (el discurso) como práctica social. Supone una concepción activa del lenguaje y del discurso porque, para nuestro caso, organizan las formas de emprender y conciliar diversas prácticas sociales que, finalmente, reproducen estructuras de desigualdad social. De manera articulada a este enfoque, el análisis recurre a la Teoría de la Valoración (Martin y White 2005); Asumimos este enfoque porque, específicamente, en este trabajo se interpretan las evaluaciones de la propia actividad emprendedora de las mujeres, las posiciones e identidades asumidas por las entrevistadas, y las formas en que negocian las relaciones sociales en las que están insertas. En concreto, observamos y analizamos discursivamente la actitud hacia la actividad emprendedora, en relación con las "tareas reproductivas" y de conciliación.

\section{Género y emprendimiento}

\subsection{El carácter performativo del género}

En este apartado sintetizamos los conceptos teóricos vinculados al análisis de género (Brunet et al. 2009a; 2009b, 2011; Brunet y Pizzi, 2011), y que fueron utilizados en la investigación empírica. Desde una perspectiva de análisis centrada en los factores del entorno de la creación de empresas, la teoría económica institucional (North 2005; Fayolle et al. 2005) plantea que el marco institucional y los valores vigentes en cada momento histórico de las sociedades condicionan la actividad empresarial. Una de las bases de dicho marco institucional es el género, en el sentido de que la división institucional y analítica entre la producción, por un lado, y la reproducción, por otro, corre pareja con la escisión en el ámbito de los espacios sociales público/privadodoméstico, así como también con la escisión entre los roles masculino/femenino. 
Estas dicotomías implicaron institucionalmente no sólo la distribución de tareas por géneros, sino la construcción diferenciada de subjetividades. Uno de los aspectos que condicionó el surgimiento de la categoría de "género" fue la comprensión de que los rasgos "masculinos" y "femeninos" asignados a los hombres y mujeres no estaban determinados por su sexo biológico, sino que estaban condicionados por factores sociales (Melero, 2010). El concepto de "género", por tanto, constituye un constructo que se diferencia del "sexo". Éste se ubica en el ámbito biológico-anatómico, mientras que el "género" se asigna al ámbito cultural y simbólico. Por tanto, se definen en función de características normativas asignadas a lo masculino y femenino, y permite la construcción de identidades y relaciones de poder (Melero, 2010). Este uso de la categoría género mantiene los dualismos entre una dimensión biológica y una dimensión social. De aquí la necesidad de plantear otro uso de dicha categoría, en el sentido de ser "una forma primaria de relaciones significativas de poder" (Scott 1990), lo que "conlleva una develación de los modos de significarse recíprocamente género y poder" (Amigot y Pujal 2009: 119). Por ejemplo, en referencia a estos modos de significarse, Butler (2002; 2006a) plantea que la orientación sexual es un ente regulado por la 'norma de género' y no es un impulso innato. Por este motivo, el deseo se encuentra regulado socialmente (Vélez-Pelligrini, 2008).

De acuerdo a la perspectiva de Butler (2001a), el sexo siempre estuvo ligado al género, en el sentido de que lo natural siempre está vinculado a lo histórico-cultural. No existiría mujer "natural" que después se transforma en una mujer socialmente subordinada, dado que la naturaleza humana es inherentemente histórica.
De este modo, Butler $(2010,2006 a)$ ha elaborado una interpretación del sexo y del cuerpo que pone en tela de juicio la oposición tradicional entre naturaleza y cultura. Ha desarrollado un complejo conjunto de ideas proponiendo que el sexo es un logro cultural con consecuencias (materiales) sobre el cuerpo. Si el género es la construcción cultural del sexo, entonces el sexo y el cuerpo son los efectos del discurso en el sentido que han sido producidos por él. Esto no significa, señala Rose (2010), que el sexo y el cuerpo son imaginados, o que son de alguna forma inventados por el lenguaje. Por el contrario, Butler (2002) defiende que el propio cuerpo adquiere un género a través de acciones reiteradas, un proceso que describe como de "performatividad". El género, en otras palabras, se torna corpóreo, y lo que pensamos como sexo es el efecto de esta práctica reiterativa o ritual. Una práctica que resulta en que el sexo sea percibido como algo totalmente "natural".

En definitiva, en el análisis nos apoyamos en estas consideraciones para destacar que entendemos el género como una dimensión performativa. Dada la discusión teórica previa, asumimos que las características identitarias del género femenino son resultado de una práctica social de reiteración colectiva (Brunet et al. 2009a, 2011; Brunet y Pizzi, 2011), y descartamos la perspectiva que vincula la identidad de género con determinaciones biológicas.

\subsection{Mujeres emprendedoras y el handicap de género}

En este sup-apartado sistematizamos los resultados de estudios previos sobre el vínculo entre la creación de empresas y género, y que constituyen el marco de referencias empíricas de nuestra investigación. 
De acuerdo al Global Enterpreneurship Monitor (GEM 2014a, 2014b), en ningún país del entorno europeo el porcentaje de mujeres ocupadas por cuenta propia supera al de los hombres. De acuerdo a estos datos, al momento de nuestra investigación el país que presenta una mayor igualdad es Portugal ${ }^{1}$, y el que refleja mayor desequilibrio es Irlanda ${ }^{2}$. Por otra parte, a nivel mundial, también los países que tienen una mayor tasa de emprendimiento femenino son los menos desarrollados (lo que indica el fuerte peso de los "emprendimientos por necesidad" entre el total de los emprendimientos por cuenta propia). La situación española, en 2014, revela que la actividad emprendedora masculina es de un $6 \%$ (disminuyendo con respecto a años anteriores), mientras que la femenina un $4 \%$ (aumentando con relación a años anteriores). La actividad emprendedora femenina es superior a países como Francia, Alemania, Dinamarca, Bélgica y Eslovenia, y se ubica en una situación similar a la de Suecia (GEM 2014a; 2014b).

En relación con los emprendimientos masculinos, los femeninos se ubican más entre los emprendimientos "nacientes" (la puesta en marcha) que entre los "nuevos" (hasta 42 meses), lo que indica un mayor tiempo de duración de los emprendimientos masculinos. Además, los emprendimientos masculinos en promedio tienen más empleados que los femeninos. Otras características relevantes para nuestros intereses dan cuenta de que los emprendimientos innovadores también prevalecen entre los hombres por sobre las mujeres, y el nivel

En Portugal, las trabajadoras por cuenta propia representan el $19,9 \%$ del total de mujeres ocupadas, y los hombres el $24 \%$ de los ocupados. educativo es superior entre los emprendedores masculinos (GEM 2014a, 2014b), generando una brecha en las posibilidades de explotar oportunidades reales de mercado.

Los análisis realizados, mayoritariamente en las Islas Británicas y norte de Europa, muestran cómo las mujeres experimentan desventajas tanto en la creación como en la gestión de las empresas (Carter 2000; Marlow 2002; Holmqvist y Sundin 2002). En relación con las empresas dirigidas por hombres, las empresas lideradas por mujeres presentan una menor capitalización, rentabilidad, concentración sectorial, así como también reciben estereotipos negativos, dado que se las asocia con metas estratégicas menos ambiciosas que las que tienen los emprendedores y directivos masculinos (Bird y Shapp 2004; Shaw et al. 2001; Rosa y Hamilton 1994). La literatura especializada destaca que estos rasgos estructurales constituyen las causas por las cuales la cantidad de empresas dirigidas por mujeres, dedicadas a la exportación, que experimentan un rápido crecimiento y que necesitan financiación bancaria, es menor que las dirigidas por hombres (Carter y Brush, 2004; Carter et al. 2006).

Por otra parte, "la necesidad de buscar fórmulas alternativas de conciliación de la vida laboral y familiar conduce a las mujeres a tener mayor propensión hacia el autoempleo que los hombres" (Brunet y Alarcón, 2005: 550). De hecho, los incentivos de los emprendimientos y autoempleo de hombres y mujeres son diferentes. La vinculación de ambos con respecto al trabajo productivo y reproductivo es desigual, en el sentido de que los hombres no incrementan su participación en el trabajo doméstico al mismo ritmo proporcional en que se incorporan las mujeres al mercado laboral. 
Las investigaciones internacionales de décadas pasadas, como las de Connelly (1992), Powell y Mainiero (1992) y Caputo y Dolinsky (1998) sugerían "que existe una correlación positiva entre el número de hijos y la probabilidad de creación de empresas por parte de mujeres, en especial durante la infancia de los mismos" (Brunet et al. 2009: 145). Con posterioridad, a partir de estos resultados, otros estudios han mostrado que los incentivos de las iniciativas de autoempleo están vinculados con las responsabilidades domésticas y las cargas familiares (Shelton 2006; Baines y Weelock 2000). En este sentido, el autoempleo tiene una valoración favorable debido a que ofrece cierta flexibilidad en la cantidad y tiempo de trabajo dedicado al mismo, así como la posibilidad eventual de trabajar en el hogar. Todo ello, en principio, favorecería una mejor conciliación laboral y familiar (Brunet y Alarcón, 2007).

No obstante, otros resultados destacan que el autoempleo tiene efectos no deseados, una mayor carga laboral, y ello repercute en una disminución del tiempo dedicado a la conciliación familiar (Hildebrandt y Williams 2003). En una investigación realizada a partir del panel de hogares de la Unión Europea con datos relativos a ocho países, Williams (2004) indica que lo que explica la sustentabilidad económica de los emprendimientos por cuenta propia es la cantidad de tiempo dedicado a dicho trabajo.

El género de las emprendedoras constituye una restricción porque éstas se ven constreñidas, en buena medida, a afrontar la doble tarea de conducir sus empresas y realizar las tareas domésticas y el trabajo reproductivo (Ferguson y Durup 1997, Marlow 1997, Brush et al. 2004). Dichas restricciones se refuerzan porque en el ámbito familiar no existen las instituciones de regulación laboral como la negociación colectiva ni agentes sociales organizados. Así, los conflictos que surgen en los hogares no son visibilizados públicamente, o como problemas de orden público. Las tareas domésticas y de cuidado no son consideradas "trabajo", a menos que las realice el personal doméstico contratado para ello (Brunet et al. 2009a, 2009b, 2011).

Para explicar el menor número de empresas dirigidas por mujeres, además de las tensiones entre la conciliación de la vida laboral y familiar, la bibliografía destaca que las posibilidades de ascenso de las mujeres directivas (asalariadas) en las estructuras de gestión de los negocios en las empresas, están condicionados por la existencia de mecanismos sociales (el "techo de cristal") que afectan sus carreras profesionales. Las directivas experimentan limitaciones a nivel de competencias, así como de la ampliación de sus capitales sociales y relacionales (Verheul et al. 2003; Weiler y Bernasek 2001; Halford y Leonard 2001). Dicho capital social se encuentra incrustado en las relaciones sociales y en las redes que surgen de las mismas, y su control favorece la supervivencia y el crecimiento de las empresas (Aldrich y Zimmer 1986; Parker 2004; Prieto 2007).

Por otra parte, con relación a las dificultades financieras adicionales derivadas de su situación de género, las mujeres asalariadas experimentan una situación de subordinación en el mercado laboral por la cual suelen disponer, en general, de salarios más bajos que los hombres y, en parte por ello, tienen menos capacidad de ahorro. Ello las limita al momento de solicitar créditos a los bancos para iniciar un emprendimiento (Carter y Kovereid 1997; Carter y Rosa 1998; Greene et al. 2000). Debido a sus menores posibilidades de capitalizarse, 
se encuentran con dificultades financieras superiores a los hombres (Marlow y Patton 2005a). Dichas dificultades de financiación condicionan a muchas emprendedoras a asumir iniciativas de baja capitalización económica, que reportan bajas tasas de rentabilidad. Por ejemplo, los emprendimientos en servicios intensivos en mano de obra, ventas al por menor, etc., constituyen casos típicos (Meager et al. 1994).

En consecuencia, las desventajas respecto a los hombres se relacionan no sólo con su vínculo con el trabajo reproductivo, sino también con su trayectoria previa como asalariadas. La combinación de los factores anteriores explica la tendencia, por parte de dichas mujeres, al establecimiento de emprendimientos de baja rentabilidad, manos de obra intensivos y sometidos a fuerte competencia de mercado. Por ejemplo, en el Reino Unido, donde existe una amplia evidencia empírica relativa a la creación de empresas por parte de mujeres, luego de tres décadas de haberse aprobado una legislación antidiscriminatoria, las mujeres continúan afrontando la mayor parte del trabajo reproductivo/doméstico, al tiempo que no se han desmantelado los mecanismos de segregación ocupacional que afecta el estatus económico de las mujeres (Maushart 2001). La forma en que se combinan estas formas de segregación ocupacional y trabajo doméstico siguen constituyendo una limitación para que las mujeres emprendedoras logren acumular credibilidad financiera y ello les facilite incorporarse de forma no precaria a la actividad emprendedora (Marlow 2002).

Para finalizar, con relación a las diferencias de género en los estilos de liderazgo, resultan relevantes las condiciones en que los hombres y mujeres lideran las organizaciones. En este sentido, se pueden mencionar brevemente los diferentes estilos de liderazgo empresarial que afectan dichas condiciones, como el transformacional, la codirección, el liderazgo en red, de corte ético (basado en valoraciones de justicia social e igualdad), y liderazgos en "empresas sociales" que no buscan la maximización del beneficio económico (Berbel, 2013). Al respecto, esta autora plantea que los cambios en los patrones culturales, para prosperar, requerirán de la inscripción de las estrategias directivas de las mujeres en redes de apoyo que favorezcan la articulación de demandas de igualdad de género.

\section{Las lógicas sociales del emprendimiento femenino}

Presentamos el análisis de los discursos sociales dominantes de las mujeres empresarias en España, basado en la investigación previa (Brunet et al. 2009a, 2009b, 2011; Brunet y Pizzi, 2011) y, a continuación, reelaboramos sus dos variantes principales, reconstruidas sobre el eje trabajo productivo/reproductivo, cuya relevancia conceptual se fundamenta en los desarrollos teóricos elaborados en el apartado anterior.

\subsection{El trabajo enraizado en las relaciones de género}

La actividad empresarial está enraizada en la vida social, y por tanto excede el ámbito de la firma o del proceso de trabajo. Esta dimensión social incluye el sistema de género. En diferentes regiones, culturas o países, así como en distintos períodos históricos, la división sexual de trabajo puede cambiar sus características. 
Lo significativo, sin embargo, consiste en que las tareas se asignen diferencialmente en función del género (Talpade, 2008). No obstante, según los contextos, pueden ser distintos los significados de la división sexual del trabajo.

La "división sexual del trabajo" supone que el trabajo de los hombres tiene un valor diferencial con respecto al trabajo de las mujeres. En la investigación de campo visualizamos unos marcados rasgos androcéntricos: las mujeres emprendedoras perciben su género como un aspecto limitante en relación al desempeño masculino en el mundo empresarial ("Las mujeres solemos ser más meticulosas, más cuidadosas, la agresividad suelen tenerla más los hombres que las mujeres. Creo que el hombre es más competitivo por naturaleza. Sí, a nivel competitivo el hombre desarrolla mejor la tarea, porque él es más competitivo y tiene mayor capacidad para pelear" -Emprendedora $7^{3}$-). Típicamente, la competitividad la encontramos relacionada con la agresividad, y asociada a lo masculino, mientras que lo femenino se vincula discursivamente con la meticulosidad y el cuidado.

En tanto que la condición de género existe aún antes de que un sujeto descubra la diferencia sexual, las subjetividades que asumen los contenidos tradicionales asociados discursivamente a lo "masculino" y "femenino" reproducen el valor diferencial atribuido al "trabajo de los hombres". Ello lo observamos en el discurso social de las entrevistadas como un rasgo repetitivo, donde vemos con claridad que los valores de "egoísmo", "agresividad", "eficiencia", asociados a la competitividad en el mundo de los negocios, se vinculan con

\footnotetext{
Informática, diseñadora de software, autónoma, ningún trabajador a su cargo, vive en pareja, 1 hijo. Cataluña.
}

referencias "masculinas", mientras que la "empatía", la "solidaridad", el compromiso con los "afectos" se vinculan con concepciones tradicionales de lo "femenino". Tomamos un ejemplo ilustrativo de este discurso social: "los hombres son más egoístas, más agresivos, más racionales", mientras que "las mujeres nos comportamos más emocionalmente, y por ello somos más solidarias y empáticas". Por ello, el siguiente relato ilustra que, en el mundo de los negocios, las mujeres "tenemos que usar la psicología masculina" (Emprendedora44). Así, las emprendedoras de oportunidad le asignan un carácter "masculino" al mundo de los negocios, y ello nos permite comprender las formas de subjetivación que hemos observado.

No se observa un discurso en el que se cuestione el monopolio masculino de los negocios, sino que ello se asume y naturaliza. Además, no se reconocen particularidades o especificidades de gestión "femenina". A modo de ilustración: ("en los negocios no existe capacidad o habilidad femenina diferente a la de los empresarios" (Emprendedora315). O bien: "la actividad profesional la hace igual un hombre $y$ una mujer; en este sentido no hay diferencias" (Emprendedora336)-. Aquella naturalización se traduce en que las diferencias masculino/ femenino se interpretan como tensión de carácter, entre personalidades "agresivas" y "empáticas".

En este sentido, observamos un discurso en el que las mujeres se pueden asemejar a los hombres en el mundo de los negocios si aumentan su

Licenciada en marketing, investigadora de mercados, autónoma, 2 trabajadores, casada, 1 hijo. Cataluña.

5 Graduada en economía, consultoría fiscal y financiera, autónoma, sin empleados a cargo, casada con 2 hijos. Valencia.

6 Abogada, autónoma, sin trabajadores a cargo, casada, 1 hijo. Valencia. 
"agresividad", dejando de lado su emocionalidad y empatía ("si tú eres una persona bastante agresiva pues puedes equipararte a un hombre que sea un poco más mediocre que tú, pero para eso necesitas tener más agresividad, en igualdad de condiciones la verdad que no" (AndalucíaEmprendedora157). Este ejemplo es ilustrativo de un discurso que plantea la necesidad de las mujeres de acercarse al "mundo masculino" de los negocios, con el fin de aumentar las probabilidades de éxito profesional.

Sin embargo, dentro de esta narrativa común que hemos analizado, observamos diferencias que podemos agrupar en dos espacios discursivos. En primer lugar, existe un discurso que refleja la orientación de los comportamientos y estrategias de las emprendedoras a partir de un compromiso con la conciliación entre la vida profesional y familiar. Al grupo que asume este discurso podemos denominarlo "emprendedoras conciliadoras".

En segundo lugar, observamos un discurso que justifica la adscripción profesional al ámbito del mercado y subordina los compromisos de la conciliación. Asimismo, incorpora en el relato valores considerados "masculinos" en relación al trabajo. A las emprendedoras que asumen este discurso las denominamos "emprendedoras no-conciliadoras". No significa que quieran ser hombres ni piensan que la actividad profesional sea "cuestión de hombres", sino que los valores que se consideran idóneos para ejercer la profesión se asocian, en el discurso, con contenidos específicos que siempre se vincularon con lo "masculino".

Las "emprendedoras conciliadoras" consideran

Licenciada en farmacia, empresaria de laboratorio clínico, autónoma, 6 trabajadores, casada, 2 hijos. Andalucía. que hombres y mujeres poseen esquemas de comportamientos directivos distintos. Asocian el estilo directivo femenino a la "maternidad", es decir, a rasgos psicológicos propios del espacio privado y la vida en el hogar. Para ilustrar: "yo creo que las mujeres mantenemos una relación diferente con los empleados que los hombres, ya que nosotras miramos más dentro del empleado. Nos preocupamos más por lo que le sucede" (Emprendedora2 ${ }^{8}$ ).

De acuerdo a la cultura patriarcal occidental, la concepción de la "maternidad" incluye a un saber-hacer vinculado a la tolerancia, la paciencia, el cuidado, la protección y el sacrificio. Constituye un discurso que no ha sido desarticulado a partir de la influencia del homo economicus como representación dominante de las prácticas económicas modernas, sino que se encuentra articulado con él en las prácticas concretas. De esta compleja articulación vemos que la percepción de la división sexual del trabajo por parte de este perfil de empresarias asume que en ámbito de los negocios existe una realidad "unisex", en el que las mujeres pueden imprimir sus matices, y el ámbito privado es uno "del que se tiene que hacer cargo la mujer" (Emprendedora $37^{\circ}$ ). De esta manera se asume la conciliación como tarea fundamentalmente femenina.

Esta dualidad discursiva se observa claramente en el siguiente relato: "yo tengo dos aparejadores que son cabezas de familia y sus mujeres no trabajan. Lógicamente les influye más lo que van

Licenciada en administración de empresas, empresaria inmobiliaria, autónoma, 2 trabajadores a su cargo, divorciada, 2 hijos. Cataluña.

9 Licenciada en administración de empresas, consultora de gestión de recursos humanos, autónoma, ningún trabajador a cargo, casada, 1 hijo. Murcia. 
a ganar en un momento determinado, mientras que las mujeres profesionales, como es mi propio caso, como comparto gastos con mi marido, no es lo prioritario ganar dinero. Lo prioritario para mí es la familia" (Emprendedora36 ${ }^{10}$ ). Existe una tensión discursiva entre el espacio laboral, de la racionalidad y la "agresividad competitiva", por un lado, y el espacio de los afectos familiares por otro.

Las tareas de conciliación, en este perfil de emprendedoras, se organizan de acuerdo a un modelo discursivo de maternidad estructurado en torno al cuidado y afecto hacia los hijos y el marido. El emprendimiento autónomo les ofrece el tiempo para la conciliación y responsabilidades familiares.

La formación de una familia constituye, para este segmento de emprendedoras "conciliadoras", un eje estructurado central, en función del cual se organizan sus actividades y horizontes económicos y familiares. En este sentido, la familia nuclear ofrece seguridad y estabilidad emocional. Es la maternidad lo que las diferencia de los hombres, "la posibilidad de tener un hijo (...) Pues esto ellos no lo tienen (...) Porque yo no quiero la pauta de trabajar como un imbécil todo el día sin ver a la familia. (...) Yo no quiero ser directiva agresiva, estresada, con 700 móviles, que no veo nunca a mis hijos" (Emprendedora18 $\left.{ }^{11}\right)$. Este ejemplo nos ilustra un discurso en el cual lo que completa su identidad social es la conciliación y su vinculación estrecha y afectiva con su familia. Como vemos, la ausencia de estos elementos afectivos ligados

10 Licenciada en administración de empresas, consultora de empresas y de formación, autónoma, 2 trabajadores, casada, 2 hijos. Murcia.

11 Licenciada en administración de empresas, consultora inmobiliaria, autónoma, 2 trabajadores, vive en pareja, 1 hijo. Andalucía. a la familia se asocia negativamente con una limitación en el desarrollo personal.

El perfil "no-conciliador" no se plantea que su objetivo prioritario, en torno al cual se subordinan las demás iniciativas, sea la formación de una familia. Por el contrario, se prioriza el trabajo profesional. Las llamamos emprendedoras "noconciliadoras" porque asumen un discurso del éxito empresarial en base a una serie de valores y actitudes que se asocian imaginariamente con lo "masculino", tal como se analizó en párrafos anteriores. La realización profesional constituye, por el contrario, el objetivo prioritario.

De las conversaciones grupales entre emprendedoras observamos que lo que denominan la "mentalidad masculina" en los negocios consiste en asociar discursivamente valores de competitividad, agresividad y eficiencia con una noción de lo masculino. Esta línea discursiva asume que los contenidos favorables para la actividad empresarial son aquellos vinculados con lo masculino. Así: "/o importante es pasárselo bien y ganar dinero", lo que se asocia con "un rol muy masculino" (Grupo de discusión de mujeres empresarias $1^{12}$ ).

Este tipo de discurso "masculinizado", según la perspectiva de estas emprendedoras,

\footnotetext{
Valencia. 9 participantes: 1) informática, autónoma, ningún trabajador, casada, 2 hijos; 2) licenciada en administración de empresas, empresaria del sector del mueble, autónoma, 2 trabajadores, soltera; 3) licenciada en administración de empresas, empresaria de servicios a empresas, autónoma, 7 trabajadores, casada, 2 hijos; 4) pedagoga, empresaria de animación infantil, autónoma, ningún trabajador, casada, 1 hijo; 5) licenciada en farmacia, empresaria de laboratorio, 5 trabajadores, casada, 3 hijos; 6) abogada, asesor laboral y fiscal, autónoma, 2 trabajadores, casada, 1 hijo; 7) pedagoga, empresaria de servicios educativos, autónoma, 4 trabajadores, casada, 2 hijos; 8) licenciada en marketing, investigadora de mercados, autónoma, ningún trabajador, casada, sin hijos; 9) licenciada en bellas artes, empresaria de arte, autónoma, 4 trabajadores, divorciada, 2 hijos.
} 
plantea una relación distante con el trabajo reproductivo, en tanto que distribuyen el tiempo vital fundamentalmente entre el trabajo profesional y el ocio, y no entre trabajo de mercado, trabajo doméstico y ocio. El trabajo doméstico se subcontrata; todas tienen "una mujer que les hace el trabajo", por lo tanto, respecto al trabajo doméstico "no tengo ningún problema" $\mathrm{y}$, respecto al trabajo de mercado, "tienes que ser más dura de lo que eres normalmente, una sonrisa no vale y tienes que estar siempre en plan borde. Poca simpatía y mucho carácter es lo que necesitas" (Grupo de discusión $\left.5^{13}\right)$. Dureza, fuerza, agresividad: asumir en la empresa una actitud "más dura de lo normal" implica posicionarse en un rol asociado discursivamente, por parte de estas emprendedoras, a lo masculino.

\subsection{La performatividad del discurso emprendedor de las mujeres}

Tanto en las emprendedoras "conciliadoras" como en las "no-conciliadoras", podemos ver la realidad de género como una construcción social que se expresa individualmente, a través de subjetividades que repiten performances normativizadas, detectada en la investigación original (Brunet et al. 2009a, 2009b). El eje discursivo femenino/masculino concibe ambos polos de manera opuesta y complementaria. Dicha complementariedad remite a que hombre y mujer constituyen un organismo completo.

Andalucía. 10 participantes: 1) licenciada en pedagogía, empresaria de ludoteca, autónoma, 4 trabajadores, casada, 1 hijo; 2) licenciada en administración de empresas, consultora de calidad, autónoma, 2 trabajadores, separada, 1 hijo; 3) licenciada en pedagogía, consultora social, autónoma, ningún trabajador, casada, 3 hijos; 4) licenciada en arquitectura, dirige un taller de arquitectura, autónoma, 4 trabajadores, casada, 2 hijos; 5) licenciada en derecho, laboralista, autónoma, casada, 1 hijo; 6) licenciada en pedagogía, empresaria de sector de restauración, autónoma, 10 trabajadores, casada, 3 hijos.
En los discursos de género de estos perfiles, dichas oposiciones y complementariedades se presentan como naturales. En este sentido, el discurso de las emprendedoras de oportunidad, que incluye ambos perfiles considerados ("conciliador" y "no-conciliador"), reproduce normas típicas; por ejemplo, la concepción de la maternidad como posición y privilegio de las mujeres.

Dentro de este discurso general, las "emprendedoras conciliadoras" organizan sus responsabilidades de conciliación el marco convencional del modelo doméstico. Una empresaria nos ilustra: "de entrada las mujeres trabajamos más. Y las madres aún más. Es la percepción que tengo. Para equipararte con los hombres debes esforzarte mucho más (...). Conozco muchas mujeres que el horario... ¿qué horario? El trabajo se hace y ¿cuántas horas le has dedicado? No tengo ni idea. Es una forma diferente de funcionar." (Emprendedora8 ${ }^{14}$ ).

La experiencia de la maternidad está asociada con construir una familia, y ello les otorga una identidad que, en el discurso social, se presenta como un destino natural para ellas. Dicha identidad remite al ámbito de las emociones y los afectos, alejado de los contenidos del ámbito del trabajo productivo. En el discurso típico de este perfil de emprendedoras no aparece la necesidad de alterar los patrones organizativos de las relaciones familiares. Como ilustración citamos las palabras de una emprendedora entrevistada: "Es muy complicad compaginar mi vida con niños. Mis horarios son intempestivos, por eso la única solución para tener hijos es tener una persona interna en casa

\footnotetext{
Ingeniera informática, diseñadora de software, autónoma, ningún trabajador, casada, 1 hijo. Cataluña.
} 
que se haga cargo de las tareas domésticas y del bebé" (Emprendora3915). La entrevistada expresa un discurso y un posicionamiento típico que implica una dinámica familiar en la que todo lo que no sea afectivo y emocional se subcontrata. En este sentido se concibe el comportamiento del marido como algo "natural, biológico", "específico de los hombres", "ellos son como son" (Grupo de Discusión de Mujeres Empresarias $3^{16}$ ).

No observamos un discurso social crítico de la realidad de género entre las emprendedoras por oportunidad. Básicamente, las emprendedoras de oportunidad "conciliadoras"

se identifican con la "clase media"; tienen trabajos y actividades propias de su categoría profesional. El trabajo doméstico (fundamentalmente la limpieza del hogar) se organiza a través de la contratación de empleadas.

Este segmento de emprendedoras asume un discurso que articula y hace compatible la dedicación a la familia y a las actividades empresariales. El mayor esfuerzo que esta compatibilización implica para las emprendedorasse justifica en clavedeganancias afectivas y emocionales, de disfrutar de la gente querida, asociado todo ello a la imagen de la familia. Sin embargo, el disfrute de la familia se

Licenciada en psicología, psicóloga profesional, ningún trabajador, casada, 1 hijo. Murcia.

16 Cataluña. 6 participantes: 1) licenciada en periodismo, free lance, autónoma, ningún trabajador, soltera , sin hijos; 2) licenciada en traducción e interpretación, traductora, autónoma, ningún trabajador, casada, 1hijo; 3) licenciada en diseño gráfico, diseñadora gráfica, autónoma, 2 trabajadores, casada, 2 hijos; 4) licenciada en ciencias políticas, consultora política, 2 trabajadores, casada, 2 hijos; 5) licenciada en arquitectura, dirige un taller de arquitectura, 4 trabajadores, casada, 1 hijo; 6) ingeniera informática, empresaria de servicios informáticos, 5 trabajadores, casada, 2 hijos. articula con la subcontratación (a otras mujeres asalariadas) de las actividades domésticas. Este mecanismo "clasista" favorece la identificación de la familia con lo afectivo/emocional que está ausente en la actividad empresarial.

Para las emprendedoras "no-conciliadoras" sus proyectos de éxito profesional no se organizan discursivamente mediante la asignación de un rol central (y complementario) al cuidado de la familia. Sus prácticas y actitudes empresariales se organizan, por el contrario, en base al discurso según el cual su éxito económico descansa en prácticas propias del género que "imitan", y no del género atribuido socialmente. Vinculan las capacidades empresarias consideradas eficientes con actitudes y disposiciones asociadas al mundo de los hombres. Este perfil de emprendedoras no vive conflictivamente las lógicas productivas y reproductivas. Se consideran mujeres autónomas y profesionales, y subordinan el rol de esposas y madres al proyecto empresarial. La prioridad vital de este perfil está puesta en el desempeño profesional.

Entre las emprendedoras "conciliadoras", la múltiple presencia en el trabajo productivo y reproductivo supone una transformación en el modelo de género. En los discursos, observamos un relato en el cual ya no son "amas de casa" sino "super-mujeres", dado que el incremento de responsabilidades consiste en asumir la conciliación de ambas esferas. Por el contrario, en las "emprendedoras no-conciliadoras" el cambio implica dejar de ser "amas de casa" para ser un "super-hombre", en el sentido de subordinar las tareas reproductivas y centrarse exclusivamente en el proyecto profesional (Brunet et al. 2009a, 2011). Sin embargo, los discursos que reconstruimos nos permiten observar que ambos perfiles, dada su posición 
social, no cuestionan su régimen de género. Inclusive se considera de forma convencional la formación de una pareja, en tanto se aceptan las posiciones desiguales de hombre y mujer dentro de la misma.

El discurso de las mujeres emprendedoras "conciliadoras" contiene elementos centrales de la performatividad hegemónica. No observamos elementos que permiten superar instituciones que objetivan las relaciones de género dominantes, como la familia y el matrimonio, que refuerzan el reconocimiento de las mujeres fundamentalmente como "madres". Este discurso percibe y configura presiones objetivas que, por un lado, les imponen un rol de trabajadoras -"hay que tener un trabajo"; "hay que tener una profesión"-, por otro lado, les imponen un rol de madres -"tener una familia, marido e hijos"; "las mujeres hemos sufrido lo que es tener que ocuparse de un niño y no poder hacerlo"- (Grupo de discusión1177).

Bajo estas presiones se detectan dos tipos de normas de referencia entre las emprendedoras "conciliadoras": la primera remite a asumir la responsabilidad de la conciliación laboral/ familiar, que denominamos "norma de responsabilización" ("yo empecé en poner mi propia empresa de formación para adaptar el horario a mis niños; error tremendo porque a los dos días tuve que meter a una niñera" (Grupo

\footnotetext{
Valencia. 8 participantes: 1) pedagoga, empresaria de taller formativo, 4 trabajadores, casada, 2 hijos; 2) licenciada en administración de empresas, empresaria del sector del mueble, autónoma, 2 trabajadores, soltera; 3) licenciada en ADE, servicios financieros, autónoma, 4 trabajadores, casada, 2 hijos; 4) informática, autónoma, ningún trabajador, casada, 2 hijos; 5) licenciada en farmacia, empresaria de laboratorio, 2 trabajadores, casada, 1 hijos; 6) abogada, asesor laboral y fiscal, autónoma, 3 trabajadores, casada, 1 hijo; 7) pedagoga, empresaria de servicios educativos, autónoma, 4 trabajadores, casada, 2 hijos; 8) licenciada en marketing, investigadora de mercados, autónoma, ningún trabajador, casada, sin hijos.
}

de Discusión de Mujeres Emprendedoras2018). La segunda norma entre las emprendedoras "conciliadoras" se refiere a la aceptación de las tareas reproductivas, y que podemos denominar "norma de resignación" frente al trabajo reproductivo. El discurso de la resignación contiene elementos que naturalizan la situación ("el hombre tiene ya una edad y que ya no hay manera de enseñarlo, porque su cabeza dice que eso es cosa de la mujer, esto es así, y así es que tú estás trabajando y estás pensando que tienes todas las cosas sin hacer, y que cuando llegues, y hayas terminado tus diez horas de trabajo, todavía tienes que ponerte a hacer cosas para el día siguiente" -Emprendedora $25^{19}$-).

El discurso de las emprendedoras "noconciliadoras" no incorpora estos dilemas. Si quieren tener éxito deben asumir los roles tradicionalmente considerados como "masculinos" y consolidarse profesionalmente en la actividad empresarial que permite el acceso a los ingresos monetarios y al reconocimiento social. Dentro de este horizonte discursivo, las prácticas vinculadas al discurso de la maternidad constituyen un obstáculo para sus oportunidades de mercado.

Las referencias discursivas a la "mujer", a la "madre", al "sexo femenino", no se vinculan con una igualdad en las responsabilidades de

18 Andalucía. 6 participantes: 1) licenciada en pedagogía, empresaria de actividades educativas, autónoma, 4 trabajadores, casada, 1 hijo; 2) licenciada en economía, empresaria inmobiliaria, autónoma, 6 trabajadores, casada, 2 hijos; 3) licenciada en derecho, asesora fiscal de empresas, autónoma, 2 trabajadores, casada, 1 hijo; 4) licenciada en administración de empresas, consultora de calidad, autónoma, 1 trabajador, casada, 1 hijo; 5) licenciada en bellas artes, organizadora de eventos, autónoma, 2 trabajadores, vive en pareja, 1 hijo; 6) licenciada en arquitectura, gestiona taller de arquitectura, autónoma, 5 trabajadores, casada, 2 hijos.

19 Murcia. Licenciada en administración de empresas, empresaria del sector turístico, autónoma, 7 trabajadores, vive en pareja, 1 hijo. 
conciliación por parte de los hombres, sino que aquellos elementos discursivos sobre lo femenino se asocian con las responsabilidades de conciliación laboral/familiar de las mujeres casadas o que conviven con pareja e hijos (Brunet et al. 2009a, 2009b, 2011). El trabajo constituye una fuente de ingresos y de autonomía económica para las mujeres, que se repite en el argumento: "si no trabajas, no ganas dinero" (Emprendedora14 ${ }^{20}$ ). No obstante, dicha autonomía no cuestiona la responsabilización por la conciliación.

Entre las emprendedoras "conciliadoras" observamos un discurso que, aunque reconoce que a los hombres les corresponde asumir tareas reproductivas y de conciliación -es lo políticamente correcto-, también refleja la realidad de la desigual carga de trabajo en este ámbito. La dominación de género, las posiciones desiguales del marido y la mujer dentro del matrimonio, se evidencian en el discurso de este tipo de emprendedoras conciliadoras. Por ejemplo, respecto a tareas de conciliación y cuidado en el hogar: "pues yo, yo lo tengo que hacer básicamente todo" (Emprendedora3721).

Los discursos analizados permiten inferir que los hombres no cargan con las mismas presiones psicológicas derivadas de aquéllas obligaciones, al tiempo que también se benefician directamente de las actividades que asumen las mujeres en el hogar. Así, el discurso conciliador se manifiesta en frases repetidas por distintas entrevistadas: "Con la empresa puedes compaginar bastante bien la vida laboral y familiar". Las responsabilidades familiares y las

\footnotetext{
Cataluña. Asesora financiera, autónoma, sin empleados, casada, 1 hijo.

21 Murcia. Fotógrafa profesional. dueña de casa de fotografías. 1 empleado a cargo, 1 hijo.
}

responsabilidades empresariales se sostienen mutuamente en el discurso de las empresarias "conciliadoras".

Al sostener unos roles de género tradicionales, las emprendedoras "conciliadoras" asumen el discurso de la supremacía masculina. Esos elementos discursivos tienen un correlato en los discursos sobre la gestión de sus emprendimientos y en la concepción del estilo de dirección femenina. Resulta significativo que "si dirigimos diferente es porque las mujeres somos como madres más conciliadoras a la hora de trabajar" (Emprendedora22). En este sentido, observamos una narrativa distinta con respecto a la gestión de las emprendedoras "no-conciliadoras", que destacan la dureza y la agresividad ("masculina") como elementos de gestión eficiente y exitosa.

Nuestro análisis nos permite visualizar un discurso social en el que el ámbito de gestión de los negocios se representa con elementos asociados a lo "masculino", y ello condiciona las características, actitudes y habilidades que se necesitan para dirigir con éxito una empresa. Un ejemplo de este discurso social es que "la mujer en los negocios hace el papel que haría el hombre, intenta imitar al hombre" (Emprendedora2 ${ }^{23}$ ).

Desde el discurso de las empresarias "conciliadoras", la actividad emprendedora constituye una solución que permite articular el trabajo que reporta ingresos con el cuidado de los hijos y familiares. En el discurso, los hombres

\footnotetext{
Andalucía. Licenciada en economía, gestora fiscal y financiera, autónoma, 2 trabajadores, casada, 1 hijo.

23 Licenciada en administración de empresas, empresaria inmobiliaria, autónoma, 2 trabajadores a su cargo, divorciada, 2 hijos. Cataluña.
} 
no aparecen implicados en las tareas de conciliación ("Un hombre no tiene la tendencia de acercar el puesto de trabajo a su casa, y la mujer si"). Sin embargo, la aceptación de esta normatividad se concilia con la valoración del proyecto profesional, aunque de forma subordinada: "no estamos dispuestas a sacrificar nuestra vida personal" (Emprendedora3624).

En el caso de las emprendedoras "noconciliadoras" que hemos analizado, la creación de su empresa constituye el vehículo de progreso y éxito individual, de acuerdo a los parámetros de la cultura dominante. Ello no se justifica por la necesidad de conciliar la vida laboral y familiar. Por el contrario, la emprendeduría constituye un medio para alcanzar el éxito en la actividad económica, que implique remuneraciones y reconocimiento social acordes a sus aspiraciones.

\section{Conclusión}

Las mujeres emprendedoras autónomas no son un grupo homogéneo con características esenciales. Responden a una marcada diversidad social e individual. Sin embargo, como vimos en la discusión teórica, comparten una socialización diferenciada y una ubicación generalmente subordinada en el mundo en relación al género (Berber, 2013).

Sus discursos son ilustrativos para comprender las diferencias de género asociadas a la actividad empresarial basada en la emprendeduría por oportunidad. Las identificaciones sociales de las emprendedoras de oportunidad parecen haber

Licenciada en administración de empresas, consultora de empresas y de formación, autónoma, 2 trabajadores, casada, 2 hijos. Murcia. sido construidas sobre la superficie discursiva de los "ideales de éxito" masculino. Identificaciones que vinculan lo "masculino" con la actividad económico-empresarial. La valoración prioritaria de las prácticas y roles maternos, por parte de las emprendedoras "conciliadoras", y su subordinación por parte de las emprendedoras "no-conciliadoras" (la maternidad como obstrucción a sus oportunidades de mercado), constituyen posicionamientos que representan las diferencias entre hombres y mujeres en términos de complementariedad, o bien se justifican como producto de diferencias naturales, biológicas.

Además, entre las mujeres emprendedoras por oportunidad autónomas que formaron parte de la muestra, no hemos registrado que participaran en ámbitos colectivos, redes, plataformas, etc., a partir de los cuales inscriban sus estrategias directivas y de conciliación en un discurso de perspectiva de género crítico e inconformista. Quizá por ello, respecto del vínculo que observamos entre la emprendeduría y la familia, registramos fundamentalmente un discurso de rasgos patriarcales y normativos, sin detectarse perspectivas inconformistas o críticas de género. No observamos un discurso elaborado que cuestione los marcos sociales existentes con respecto al género, sino que éstos se toman como dados. Por ello, los discursos de este segmento de emprendedoras presentan el género como diferencia, y no como un factor asociado a la organización de la desigualdad social y de relaciones de poder.

Los resultados de esta investigación tienen, por el momento, un alcance acotado a la realidad española, y se suman al conocimiento existente reflejado en la revisión de la literatura, que excede la situación de España y abarca, incluso, 
Latinoamérica. Además, nuestra investigación ha abierto otras líneas de análisis y nos ha permitido plantear nuevos problemas y preguntas. Particularmente, resulta relevante comprobar si la tipología de mujeres emprendedoras por oportunidad que hemos analizado (conciliadoras y no-conciliadoras) nos puede permitir explicar diferentes resultados económicos de los emprendimientos. Por ejemplo, ¿qué relación existe entre ambos tipos de emprendedoras y los resultados empresariales de su actividad? Más específicamente, las emprendedoras "no-conciliadoras", que por definición tienen una dedicación más intensiva a su trabajo, ¿presentan mejores resultados empresariales que las emprendedoras "conciliadoras"? O bien, la orientación "conciliadora" ¿ejerce algún tipo de influencia específica sobre la actividad empresarial que se traduzca en resultados económicos o de gestión? Todo ello demanda llevar a cabo nuevos estudios, y la posibilidad de ofrecernos resultados novedosos y acumulativos sobre la actividad emprendedora y su vinculación con la realidad de género.

\section{Bibliografía}

Acs, Z. et al. 2005. Global entrepreneurship Monitor. Executive Report 2004. Babson Park, Babson College and London Business School.

Aldrich, H. y Zimmer, C. 1986. "Entrepreneurship through social networks". The Art Science of Entrepreneurship. Sexton, D. y Smillor, R. (eds.). Cambridge, Ballinger.

Amigot, P. y Pujal, M. 2009. "Una lectura del género como dispositivo de poder". Sociológica 24 (70): 115-152.

Ardchivili, A., Cardozo, R. y Sourav, R. 2003. "A theory of entrepreneurial opportunity identification and development". Journal of Business Venturing 18: 105-123.

Baines, S. y Wheelock, J. 2000. "Work and employment in small business: Perpetuating and challenging gender traditions". Gender, Work and Organization 1 (7): 45-56.

Berbel, S. 2013. Directivas y empresarias. Mujeres rompiendo el techo de cristal. Madrid: Aresta.

Bird, S. y Sapp, S. 2004. "Understanding the gender gap in small business success: Urban and rural comparisons". Gender and Society 18 (1): 5-28.

Brunet, I. y Alarcón, A. 2005. "Análisis comparativo de los factores condicionantes de la creación de empresas en los ámbitos rural y urbano". Revista Universitaria de Ciencias del Trabajo 6: 547-573.

2007. "Género y ocupación por cuenta propia. Análisis a través de dos studios en los ámbitos rural y urbano de Cataluña". RIPS 6 (2): 117-129.

Brunet, I. et al. 2009a. Género y creación de empresas. Efectos de la división sexual del trabajo sobre la creación de empleo por cuenta propia. Madrid: Instituto de la Mujer.

Brunet, I., Belzunegui, Á. y Valls, F. 2009b. Gènere i creació d'empreses a Catalunya. Tarragona, URV.
2011. L'esperit empresarial des de la perspectiva de gènere". Papers 96 (3): 937-957

Brunet, I. y Pizzi, A. 2011. "Dominaciones interrelacionadas (de clase, género, etc.)". Papeles del CEIC 71: 1-30.

Brunet, I. y Santamaría, C. 2013. La iniciativa emprendedora y la innovación. México: Fundación Educación Superior - Empresa.

Brush, C. et al. 2004. Clearing the hurdles: Women building high-growth businesses. Upper Saddle River, Prentice Hall.

Butler, J. 2002. Cuerpos que importan: Sobre los límites materiales y discursivos del "sexo". Buenos Aires: Paidós. 2001a. El género en disputa. México: Paidós.

Cátedra. 2001b. Mecanismos psíquicos del poder. Madrid:

2006a. Deshacer el género. Barcelona: Paidós.

2006b. Vida precaria: el poder del duelo y la violencia. Buenos Aires: Paidós.

Paidós. 2010. Marcos de guerra. Las vidas lloradas. Barcelona:

Caputo, R. y Dolinsky, A. 1998. “Women's Choice to Pursue Self-Employment: The Role of Financial and Human Capital of Household Members". Journal of Small Business Management Julio: 8-17.

Carter, S. y Kolvereid, L. 1997. Women starting new businesses: The experience in Norway and the United States. OECD Conference on Women Entrepreneurs in Small and Medium Enterprises: A Major Force in Innovation and Job Creation. April, Paris.

Carter, S. y Rosa, P. 1998. "The financing of male-and femaleowned businesses". Entrepreneurship \& Regional Development 10: $225-241$.

Carter, S. 2000. "Gender and enterprise". Enterprise and small 
business: Principles, practice and policy. Carter, S. y Jones-Evans, D. (eds.). Londres, Prentice Hall.

Carter, S. y Brush, C. 2004. "Gender". Handbook of entrepreneurial dynamics: The process of business creation. Gartner, W et al. (Comps.) London: Sage Publications.

Carter, S. et al. 2006. "Gender, entrepreneurship and business finance: Investigating the relationship between banks and entrepreneurs in the UK", Women Entrepreneurs and Their Business: A Global Research Perspective. Brush, C. et al. (eds.). Cheltenham: Edward Elgar.

Comisión de las Comunidades Europeas. 2003. Libro Verde: El espíritu empresarial en Europa. Bruselas: Dirección General de la Empresa.

Connelly, R. 1992. Self-employment and Providing Child Care. Demography 29 (1): 17-29.

Fairclough, N. 2003. "El análisis crítico del discurso como método para la investigación en ciencias sociales". Métodos de Análisis Crítico del Discurso. Barcelona: Gedisa.

Fayolle, A., Kyrö, P. y Ulijn, J. 2005. Entrepreneurship Research in Europe. Outcomes and Perspectives. Cheltneham: Edward Elgar.

Ferguson, F. y Durup, J. 1997. "Work-family conflict and entrepreneurial women: A literature review". Journal of Small Business and Entrepreneurship 14 (1): 46-57.

GEM . 2014a. Informe Global Entrepreneurship Monitor. España 2013. España: CISE.

GEM . 2014b. Global Entrepreneurship Monitor. 2013 Global Report. Massachusetts, USA, Babson College.

Giró, J. 2005. El género quebrantado. Madrid: Catarata.

Greene, $\mathrm{P}$ et al. 2003. Women entrepreneurs: moving front and center. An overview of research and theory. Coleman White Paper.

Halford, S. y Leonard, P. 2001. Gender, power and organisations. Basingstoke: Palgrave.

Hildebrand, V. y Williams, D. 2003. Self-employment and Caring for Children. IRISS-C/I Working Paper 2003-06, EPS/ INSTEAD, Differdange, Luxemburgo.

Holmquist, C. y Sundin, E. 2002. "Women as entrepreneurs in Sweden: Conclusions from a survey". Frontiers of Entrepreneurship Research. Wellesley: MA, Babson College.

Marlow, S. 1997. "Self employed women -new opportunities, old challenges?" Entrepreneurship \& Regional Development 9: 199-210.

2002. "Women and self-employment: Apart of, or apart from, theoretical construct". International Journal of Entrepreneurship and Innovation 3 (2): 83-91.

Marlow, S. y Patton, D. 2005a. "The financing of small businesses: Female experiences and strategies". International Handbook of Women and Small Business Entrepreneurship. Davidson, M. y Fielden, S. (eds.). Cheltenham, Edward Elgar. (2005b). "All credit to Men? Entrepreunership, Finance and Gender". Entrepreneurship Theory and Practice 29: 717-735.
Martin, J. y White, P. 2005. The language of evaluation: Appraisal in English. Hampshire y New York: Palgrave Macmillan.

Maushart, S. 2001. Wife work: What marriage really means for women. Londres: Bloomsbury.

Meager, N. et al. 1994. Self employment and the distribution of income. IMS. Report 270. Brighton.

Melero, A. 2010. "Reivindicar la igualdad de mujeres y hombres en la sociedad: una aproximación al concepto de género". Barataria 11: 73-83.

Moriano, J. 2005. El perfil psicosocial del emprendedor. Madrid: Consejo Económico y Social. Colección Estudios 186.

North, D. 2005. Understanding the process of economic change. Princeton: University Press.

Parker, S. 2004. The Economics of Self-Employment and Entrepreneurship. Cambridge: Cambridge University Press.

Powell, G. y Mainiero, L. 1992. "Cross-currents in the river of time: Conceptualizing the complexities of women's careers". Journal of Management 18: 215-238.

Prieto, C. 2007. Trabajo, género y tiempo social, Barcelona: Editorial Hacer.

Rosa, P. y Hamilton, D. 1994. "Gender an ownership in UK small firms". Entrepreneurship: Theory \& Practice 18 (3): 11-28.

Rose, S. 2010. ¿What is gender history? Cambridge, Polity Press.

Scott, J. 1990. "El género: Una categoría útil para el análisis histórico". Historia y Género: Las mujeres en la Europa moderna y contemporánea. Amelang, J. y Nash, M. (eds.) El Magnánimo.

Shaw, E. et al. 2001. Unequal entrepreneurs: Why female enterprise is an uphill business. Londres: The Industrial Society.

Shelton, L. 2006. "Female entrepreneurs, work-family interface". Journal of Small Business Management 44 (2): 285297.

Talpade, C. 2008. "Bajo los ojos de Occidente: academia feminista y discursos coloniales". A study of women owned businesses in the Dallas/Fort Metrople Suárez, L. et al. (eds.). Texas Woman's University, School of Management 4.

Van Dijk, T. 2000. El discurso como interacción social. Barcelona: Gedisa. . 2009. Discurso y poder. Barcelona: Gedisa.

Vélez-Pellegrini, L. 2008. Minorías sexuales y sociología de la diferencia. Gays, lesbianas y transexuales ante el debate identitario. Madrid: Montesinos.

Verheul, I., Uhlander, L. y Thurik, Roy. 2003. Business accomplishments, gender and entrepreneurial self-image, SCALES (Scientific Analysis of Entrepreneurship and SMEs). Paper N200312: EIM Business and Policy Research.

Weiler, S. y Bernasek, A. 2001. "Dodging the glass ceiling? Networks and the new wave of women entrepreneurs". The Social Science Journal 38: 85-103.

Williams, F. 2004. Rethinking Families. Londres: Calouste Gulbenkian Foundation. 